\title{
Bitter Taste in Chicken and its Implication on Nutrition
}

\author{
Enayatullah Hamdard ${ }^{1}$, Ahmadullah Zahir ${ }^{1,2}$, Babrak Karwand ${ }^{3}$, Zhicheng Shi ${ }^{1}$ and Fangxiong Shi ${ }^{1 *}$ \\ ${ }^{1}$ College of Animal Science and Technology, Nanjing Agricultural University, China \\ ${ }^{2}$ College of Food Science and Technology, Nanjing Agricultural University, China \\ ${ }^{3}$ Faculty of Veterinary Science, Kunduz University, Afghanistan \\ *Corresponding author: Fangxiong Shi, College of Animal Science and Technology, Nanjing Agricultural University, China
}

\begin{tabular}{|c|c|}
\hline ARTICLE INFO & ABSTRACT \\
\hline Received: 幽 September 22, 2019 & \multirow{4}{*}{$\begin{array}{l}\text { Bitter taste has evolved largely as a mechanism to identify nutritious foods and is } \\
\text { important for detecting nutritionally relevant food and considered as a warning signal } \\
\text { prior to ingestion against toxic/poisonous, harmful and toxic compounds. It has been } \\
\text { argued that birds have a lower taste perception compared to mammals due to their } \\
\text { low taste bud numbers present in their body. In chicken predicted taste genes for bitter, } \\
\text { sweet, umami, salt, calcium and lipids are present and preliminary data indicate that } \\
\text { bitter taste receptor repertoire is small in chickens, but in some bird species it's as large } \\
\text { as in mammals. Recently novel findings have potential to bring important innovations } \\
\text { to the practice of poultry nutrition such as optimize the use of amino acids and fats, } \\
\text { phosphorus excretion and use of alternative feedstuffs manipulation of feed intake. In } \\
\text { conclusion, avian taste is intimately associated to nutrient sensing and consequently } \\
\text { influence poultry nutrition practices. }\end{array}$} \\
\hline Published: 慧 November 08, 2019 & \\
\hline $\begin{array}{l}\text { tation: Enayatullah Hamdard, Ahmadul- } \\
\text { Zahir, Babrak Karwand, Zhicheng } \\
\text { i, Fangxiong Shi. Bitter Taste in Chick- } \\
\text { and its Implication on Nutrition. Bi- } \\
\text { ned J Sci \& Tech Res 22(4)-2019. BJSTR. } \\
\text { S.ID.003787. }\end{array}$ & \\
\hline & \\
\hline
\end{tabular}

\section{Introduction}

In the animal kingdom taste perception is critical biological mechanism for disrupting food and water intake selection prior to its consumption. It empowers chickens to distinguish productive and beneficial nutrients considering essential and vital foods such as carbohydrates and amino acids from nonbeneficial nutrients such as toxic, poisonous and harmful compounds. For elucidating the evolution of taste sense from birds to mammals, its crucial to improve the efficiency of poultry feeding through preferable feeding practices and clarify the sense of 5 basic tastes in chicken respectively [1]. In chicken bitter is the most crucial biological taste disruptor, it provokes chickens against consuming hazard and destructive foods prior to ingestion and therefore considered as a cautionary indicator. which tells chicken which prospective foods are nutritious, poisonous/toxic, harmful and these taste indications have been linked/associated to food identification, recognition and avoidance $[1,2]$.

The T2R is sub family of bitter taste, consisting of approximately 2040 genes identified in mammals recently [3]. In animals bitter taste perception is mediated by super family of guanine nucleotide binding regulatory protein (G-Protein) and G-Protein coupled receptor (GPCR) family - the taste 2 receptors (T2R) and their effectors downstream relevant proteins, although umami and sweet tastes receptors are mediated by GPCR family taste 1 receptors (T1R) and its respective proteins. Despite the gustatory organs, the taste receptors and their downstream proteins have been investigated in extra gustatory tissues in chickens and mammals, the recently investigated organs are lungs, spleen, heart, kidneys and bursa fabricus $[1,4]$. Currently the researchers confirmed that the chicken genome owing only three (3) bitter taste receptors termed ggTas2R1, ggTas2R2 and ggTas2R7, whereas the umami and sweet receptors are ggTas1R1 and ggTas1R3 hetrodiamter but absence of sweet receptor ggTas1R2.

Considering the previous studies, in chicken taste buds' development begins prenatally in variation of shape, size and number of buds at particular area present in chicken and completed by embryonic day 19 [5]. Despite to humans, excessive number $(69 \%)$ of chicken taste buds are positioned mainly in the upper palate and decisive number (29\%) are located in lower palate, whereas a minor percentage $(2 \%)$ are originate in the posteroventrolateral region of the anterior tongue correspondingly [5,6]. In comparisons to humans' beings and other mammals, birds were identified to have few taste buds among them. For example, in 
humans approximately 9000 taste buds are confirmed, in chicken between 250350 taste buds are confirmed, while pigeons owing only 3775 respectively [7]. Further, in Avian Species aforementioned number of taste buds mostly found around salivary glands in the soft epithelium of the palate, the base of tongue and the pharynx [8]. However, an insufficient number of investigations have been done to bitter taste in chicken and it has been addressed that chicken have a well-developed sense of taste but only three bitter taste receptors have been recently identified [9]. Thus, deliberate identification of bitter taste receptors and their corresponding detection thresholds is crucial for addressing the potential effects on chicken feeding performance/behavior and desired improvement on growth performance.

Remarkably, as mentioned earlier, the chicken genome owing only three (3) bitter taste receptors. The presence of minimum number of bitter taste receptors, enables the chicken a symbolic and minimalistic model for understanding of vertebrate teste perceptions and perspectives [7]. With the intermittent inflation in the cost of animal feed and higher standards of livestock products, people endeavor to discover novel feed additives and effective alternatives to substitute traditional feeding [10]. Efforts have been made to extract incredible numbers of potential additives from natural plants, and they often display bitter taste. After the identification of taste buds' throughputs in white leghorn stains, it found the lowest compare to the Rhode Island Red strain with highest number of taste buds in the broiler chickens accordingly. Meanwhile, the number of confirmed taste buds present in oral cavity were well correlated with bitter taste sensitivity. Therefore, its suggested that the number of taste buds is a crucial vital influencing factor in the recognition of bitter taste perceptions and maybe valuable in selecting appropriate feed stuffs for chickens feeding [11] Table 1 and Figure 1.

Table 1: Number of Taste Buds, Family 1 (T1R) and 2 (T2R) Taste Gene Repertoire in Birds Compared to Humans and Pigs.

\begin{tabular}{|c|c|c|c|c|}
\hline & Scientific Name & Number of Taste Buds & T1R Genes & Number of T2R Genes \\
\hline Human & (Homo sapiens) & 7902 & $\mathrm{~T} 1 \mathrm{R} 1 / 2 / 3$ & 25 \\
\hline Pig & (Sus scrofa) & 19,904 & $\mathrm{~T} 1 \mathrm{R} 1 / 2 / 3$ & $10^{\mathrm{a}}$ \\
\hline Blue Tit & (Cyanistes caeruleus) & 24 & - & - \\
\hline Chicken & (Gallus Gallus) & & $\mathrm{T} 1 \mathrm{R} 1 / 3$ & 3 \\
\hline Broiler & & 312 & - & - \\
\hline White Leghorn & & 192 & - & - \\
\hline Rhode Island & & 253 & - & - \\
\hline Duck & (Anatidae spp.) & 375 & - & - \\
\hline Parrot & (Psittacidae spp.) & 350 & - & - \\
\hline Pigeon & (Columba livia domestica) & 56 & - & - \\
\hline Quail & (Coturnix japonica) & 62 & - & - \\
\hline Sparrow & (Zonotrichia albicollis) & - & - & 18 \\
\hline European starling & (Sturnus vulgaris) & 200 & - & - \\
\hline Turkey & (Meleagris gallopavo) & - & $\mathrm{T} 1 \mathrm{R} 1 / 3^{\mathrm{a}}$ & $2 a$ \\
\hline Zebra finch & (Taeniopygia guttata) & - & $\mathrm{T} 1 \mathrm{R} 1 / 3^{\mathrm{a}}$ & 7 \\
\hline
\end{tabular}

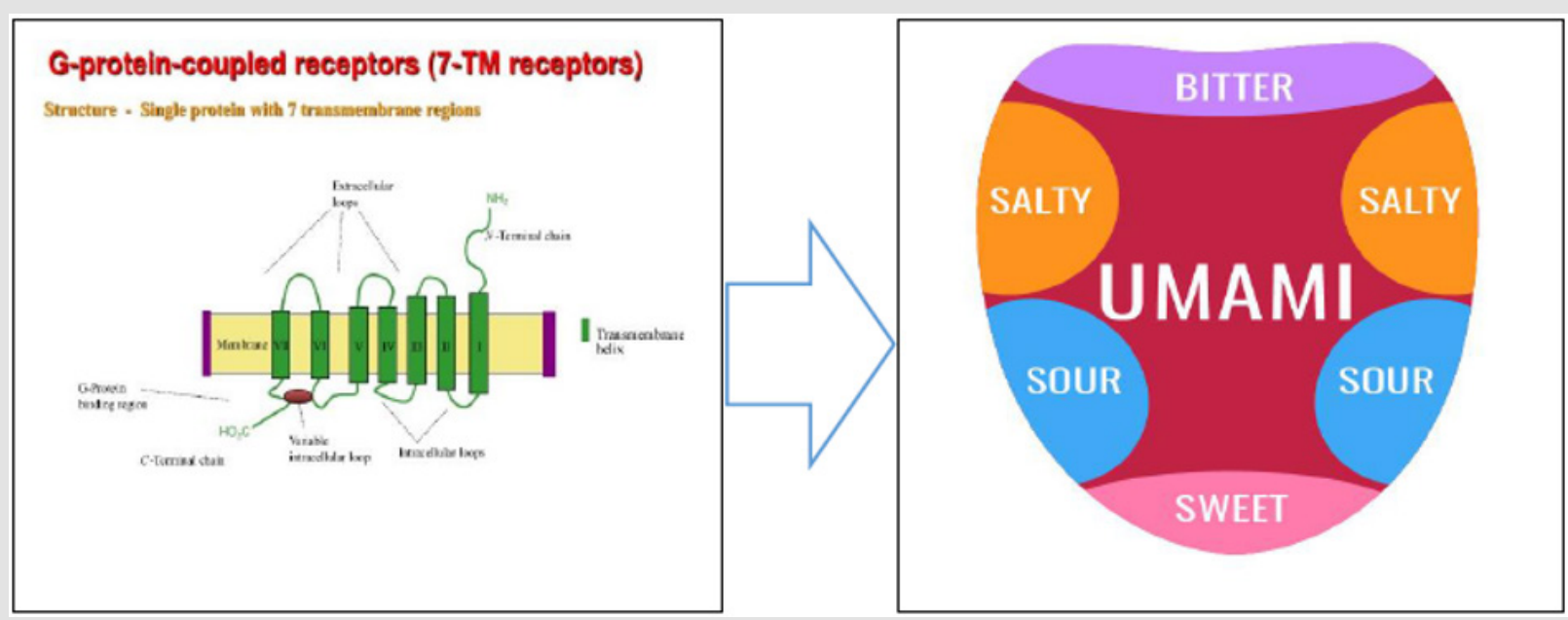

Figure 1: The chemical structure of GPCR and distribution anatomical origins of taste in tongue 


\section{Bitter Compounds Database}

Identification of bitter taste and its poisonous compounds aversion is thought to protect the organism against ingestion, which are commonly bitter. Interestingly, bitter taste receptors expressions are not limited to the gastrointestinal tract, but it's also expressed in extra oral tissues, such as lungs, heart, spleen, kidney and bursa fabricus of Chinese Fast Yellow Chicken. These expressions demonstrating that they may expose a critical role in digestive and metabolic processes [12-15]. Bitter DB database includes over 550 compounds, that were reported to taste bitter to humans, available at http://bitterdb.agri.huji.ac.il/dbbitter. php. The intention of Bitter DB is to enable studying the chemical features of various compounds associated with bitterness [16].

\section{Conclusion and Future Perspectives}

The avian taste system consists of a group of nutrients sensors evolved to evaluate the nutritional quality and content of foods. Chicken seem to be highly sensitive against bitterness and bitterness sensitivity decreased subsequently. Therefore, a sense of tasting play potential role in nutrient sensing and accepting food prior to ingestion and can influence the growth performance and physiological functions. All these novel findings open a new window for demonstration of new feedstuffs and would contribute toward improvement of chicken innovative and alternative feeds in poultry industries.

\section{References}

1. Yoshida Y, Kawabata Y, Kawabata F, Nishimura S, Tabata S (2015) Expressions of multiple umami taste receptors in oral and gastrointestinal tissues, and umami taste synergism in chickens. Biochemical and biophysical research communications 466(3): 346-349.

2. Hamdard E, Shi Z, Lv Z, Zahir A, Wei Q, et al. (2019) Denatonium Benzoate-Induces Oxidative Stress in the Heart and Kidney of Chinese Fast Yellow Chickens by Regulating Apoptosis, Autophagy, Antioxidative Activities and Bitter Taste Receptor Gene Expressions. Animals 9(9): E701.

3. Shi P, J Zhang (2005) Contrasting modes of evolution between vertebrate sweet/umami receptor genes and bitter receptor genes. Molecular biology and evolution 23(2): 292-300.

\section{ISSN: 2574-1241}

DOI: 10.26717/BJSTR.2019.22.003787

Fangxiong Shi. Biomed J Sci \& Tech Res

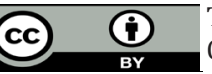

This work is licensed under Creative

Commons Attribution 4.0 License

Submission Link: https://biomedres.us/submit-manuscript.php
4. Cheled-Shoval SL, Reicher N, Niv MY, Uni Z (2017) Detecting thresholds for bitter, umami, and sweet tastants in broiler chicken using a 2-choice test method. Poultry science 96(7): 2206-2218.

5. Ganchrow JR, D Ganchrow (1987) Taste bud development in chickens (Gallus gallus domesticus). The Anatomical Record 218(1): 88-93.

6. Cheled-Shoval SL, S Druyan, Z Uni (2015) Bitter, sweet and umami taste receptors and downstream signaling effectors: Expression in embryonic and growing chicken gastrointestinal tract. Poultry science 94(8): 19281941.

7. Cheled-Shoval S, Behrens M, Korb A, Di Pizio A, Meyerhof W, et al. (2017) From cell to beak: In-vitro and in-vivo characterization of chicken bitter taste thresholds. Molecules 22(5): 821.

8. Kurosawa T, Sueo Niimura, Seiji Kusuhara, Kazuo Ishida (1983) Morphological studies of taste buds in chickens. Japanese Journal of Zootechnical Science (Japan) 54(9): 502-510.

9. Clark L, J Hagelin, S Werner (2015) The chemical senses in birds, in Sturkie's avian physiology. Elsevier: 89-111.

10. Suresh G, Das RK, Kaur Brar S, Rouissi T, Avalos Ramirez A, et al. (2018) Alternatives to antibiotics in poultry feed: molecular perspectives. Critical reviews in microbiology 44(3): 318-335.

11. Kudo Ki, Shiraishi J, Nishimura S, Bungo T, Tabata S (2010) The number of taste buds is related to bitter taste sensitivity in layer and broiler chickens. Animal science journal 81(2): 240-244.

12. Meyer D, M Kare (1986) Sense organs, in Avian Physiology. Springer: 2952.

13.Zhang SC, G Hilmas, W Fahrenholtz (2006) Pressureless densification of zirconium diboride with boron carbide additions. Journal of the American Ceramic Society 89(5): 1544-1550.

14. Roura E, MW Baldwin, K Klasing (2013) The avian taste system: Potential implications in poultry nutrition. Animal Feed Science and Technology 180(1-4): $1-9$.

15. Hamdard E, Lv Z, Jiang J, Wei Q, Shi Z, et al. (2009) Responsiveness Expressions of Bitter Taste Receptors Against Denatonium Benzoate and Genistein in the Heart, Spleen, Lung, Kidney, and Bursa Fabricius of Chinese Fast Yellow Chicken. Animals 9(8): E532.

16. Wiener A, Marina Shudler, Anat Levit, Masha Y Niv (2011) Bitter DB: a database of bitter compounds. Nucleic acids research 40(D1): D413-D419.

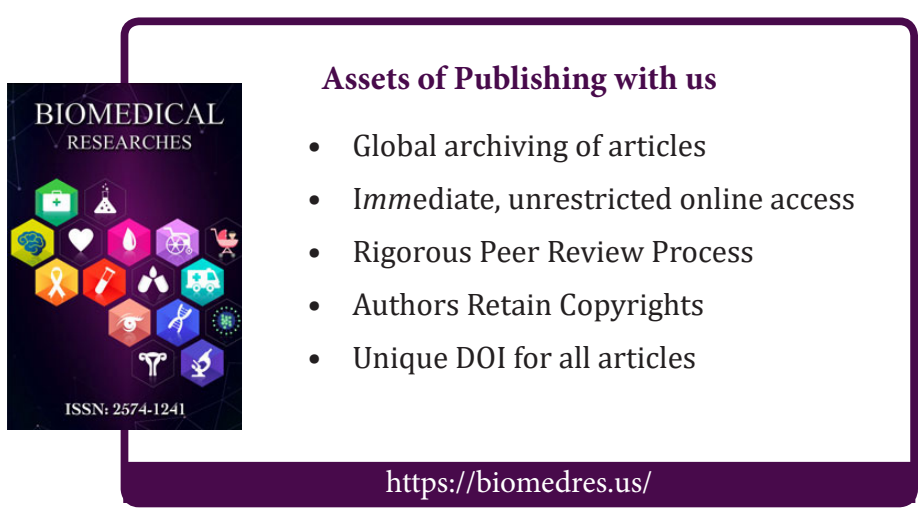

